\title{
Inhomogeneous stationary states of two-dimensional magnetofluids
}

\author{
By ROLAND CALINON
}

Département de Physique, Ecole Polytechnique Fédérale de Lausanne, CH-1007 Lausanne, Switzerland

\section{AND DANILO MERLINI†}

Department of Electrical Engineering, Northeastern University, Boston, MA, and Fakultät für Physik, Universität Bielefeld

(Received 2 June 1980 and in revised form 3 November 1982)

We discuss ensemble inhomogeneous states of general two-dimensional magnetofluids. A subclass of approximate statistical inhomogeneous states of the model equation is constructed using the principle of maximum entropy. The fields are shown to satisfy generalized nonlinear Poisson equations and some limiting cases are solved analytically. The method is then applied to a pseudo-three-dimensional model of an electromagnetic filamentation instability. The results illustrate the general role of the global constants of the motion on the nature of the statistical profiles for the fields describing the most probable states in magnetofluids.

\section{Introduction}

The purpose of this paper is to exhibit a class of approximate statistical inhomogeneous states for general two-dimensional magnetofluids and to solve analytically some special limiting cases and thus identify some general properties of the statistical profiles for the fields. The treatment is adapted here to the case of open boundary conditions and the present paper follows a first work on the subject (Calinon \& Merlini 1979) in which homogeneous stationary statistical states (solutions of the associated Hopf equation) were investigated by explicit use of the Fourier method. It should be recalled here that, besides their intrinsic interest, states of two-dimensional fluids are of importance since they appear sometimes as a useful approximation to the more complex three-dimensional situation; a typical example is the two-dimensional approximation of drift-wave turbulence recently proposed (Hasegawa \& Mima 1978). We are here concerned with the investigation of states of the fluids defined as those statistical states which are statistical solutions of the basic equations governing the flow of the magnetofluid and which, in addition, have maximum entropy. An example of these has been given recently (Montgomery, Turner \& Vahala 1979) where the most probable states of two-dimensional magnetohydrodynamics with poloidal magnetic field were investigated. The method bears some similarity to the known one in the statistical mechanics of point particles where inhomogeneous profiles for the

$\dagger$ Present address : Fakultät für Mathematik, Ruhr-Universität Bochum, 4630 Bochum, Germany. 
one-body correlation function are computed in the mean field limit. In magnetofluids there exists, in general, an infinite number of states to be investigated and we pay attention here to approximate stationary solutions of the basic equation which have the property that statistical moments factorize and which are selected within the whole class by means of an application of the principle of maximum entropy. The result also exhibits special statistical solutions of the equations which are associated with the law of conservation of a few global constants of the motion; an application of the elementary method of variational calculus to continuous distributions results in a class of nonlinear Poisson equations, and numerical methods are necessary. In this work we pay more attention to some limiting cases where a simple analytical solution may be given and studied in detail so that the effect of the constants of the motion on the nature of the profiles for the fields is immediate.

The paper is organized as follows. In $\S 2$ we first define the model equation of general two-dimensional magnetofluids which include four different situations (Euler fluid, magnetohydrodynamics with pure toroidal and pure poloidal magnetic fields and two-dimensional drift-wave turbulence) and set out the problem of stationary states. The principle of maximum entropy is applied with a detailed treatment of the Euler case, for which an analytical solution is also constructed $(\S 3)$. The method is then applied to the drift-wave equation of Hasegawa \& Mima (1978) and to two-dimensional magnetohydrodynamics; in the case of a pure toroidal magnetic field an analytical solution is given. We then proceed in $\$ 4$ with a pseudo-three-dimensional model describing the evolution of an electromagnetic filamentation instability, and in $\$ 5$ we treat in detail a stationary state of a one-dimensional fluid without neglecting triplets terms. In $\S 6$ we briefly give our conclusions.

\section{Two-dimensional magnetofluids: model equation and stationary states}

The basic equations describing the flow of two-dimensional magnetofluids, which we shall first discuss, are given by

$$
\begin{gathered}
\frac{\partial}{\partial t} \Delta \phi+\nabla^{\perp} \phi . \nabla \Delta \phi=\nabla^{\perp} a . \nabla \Delta a+\lambda^{2} \phi, \\
\frac{\partial a}{\partial t}+\nabla^{\perp} \phi . \nabla a=0,
\end{gathered}
$$

where $\phi$ and $\alpha$ are two scalar fields (the potentials) which depend only on $(x, y)$ co-ordinates and are written as three-dimensional vectors: $\phi=(0,0, \phi(x, y))$ and $a=(0,0, a(x, y))$. Equations (1) and (2) describe four different and strictly twodimensional situations. If $\lambda=0$ and $a \equiv 0,(1)$ describes the Navier-Stokes fluid (Euler Equation), while, if $a \equiv 0,(1)$ describes the evolution of the potential $\phi$ of a drift-wave turbulence model in the low-beta limit (Hasegawa \& Mima 1978); if $\lambda=0$ only, (1) and (2) describe two-dimensional magnetohydrodynamics with a pure poloidal field ( $\mathrm{B}=\left(B_{1}(x, y), B_{2}(x, y), 0\right)$; the pure toroidal case is obtained by setting $a=B_{z}$, with $B_{z}(x, y)$ being the magnetic field pointing in the $z$ direction perpendicular to the velocity field $u=\nabla^{\perp} \phi$. (Notice that for any scalar 
field $\xi=(0,0, \xi(x, y)), \nabla^{\perp} \xi=(-\partial \xi / \partial y, \partial \xi / \partial x, 0)$.) Let us introduce a bracket \langle\rangle , which refers to a statistical average associated with some probability measure for the fields $\phi, a$, denoted by $\mu_{\phi, a}$; this is a stationary measure, usually of Gibbsian type and constructed with the constants of the motion of (1) and (2) which have a direct physical meaning. The method consists usually in expanding any vector or scalar field $\xi$ by means of a set of stationary states; for example, if $a=\lambda=0$, $\phi$ is written as $\phi=\Sigma_{i} a_{i} \phi_{i}$, where the set $\left\{\phi_{i}\right\}$ is usually given by $\Delta \phi_{i}=\lambda_{i} \phi_{i}$, i.e. a particular set of stationary states satisfying the condition $\Delta^{\perp} \phi_{i} \cdot \nabla \Delta \phi_{i}=0$ in (1), and where $\left\{a_{i}\right\}$ are the random coefficients appearing in $\mu_{\phi}$. For periodic boundary conditions, $\phi_{i}=e^{i \mathbf{k} . \mathbf{x}}$ (Montgomery \& Fyfe 1976; Calinon \& Merlini 1979), while for open boundary conditions $\phi_{i} \equiv \phi_{n n}=e^{i m \theta} J_{n}(r)$ (Salmon et al. 1976; Montgomery \& Vahala 1979). The results are based on the assumption of a non-trivial kind of completeness of the set $\left\{\phi_{i}\right\}$. For the Euler flow in two dimensions, this problem has been rigorously solved only recently (Albeverio, Ribeiro de Faria \& Høegh-Krohn 1979; Albeverio \& Høegh-Krohn 1979). It should be mentioned that a more general class of stationary states $\left\{\phi_{i}\right\}$ of (1) with $a \equiv 0$ is given by those such that $\Delta \phi_{i}=g\left(\phi_{i}\right)$, where $g$ denotes any (in general) nonlinear functional of $\phi_{i}$. In fact, if

$$
\Delta \phi_{i}=g\left(\phi_{i}\right), \quad \Delta^{\perp} \phi_{i} . \nabla \Delta \phi_{i}=\left(\delta g / \delta \phi_{i}\right) \nabla^{\perp} \phi_{i} . \nabla \Delta \phi_{i}=0 ;
$$

then $\partial \phi_{i} / \partial t \equiv 0$ in (1) and $\phi_{i}$ is a stationary state. On the other hand if one is interested in stationary statistical states for the fields $(\phi, a)$ such that

$$
(\partial / \partial t) \Delta\langle\phi\rangle=(\partial / \partial t) \Delta\langle a\rangle=0
$$

in (1) and (2) (with respect to some probability measure $\mu_{\phi, a}$, invariant under time evolution), then the averaged fields $\phi, a$ should satisfy the conditions

$$
\begin{gathered}
\left\langle\nabla^{\perp} \phi . \nabla \Delta \phi\right\rangle=\left\langle\nabla^{\perp} a . \nabla \Delta a\right\rangle, \\
\left\langle\nabla^{\perp} \phi . \nabla a\right\rangle=0 .
\end{gathered}
$$

A class of approximate (not necessarily stationary) statistical solutions will be determined in the next section. We will carry out in detail the pure Euler case; with minor modification, the method applies to the general case and the resulting equations are given.

In the Euler case, the basic equations are given by

$$
\begin{gathered}
\frac{\partial \mathbf{u}}{\partial t}+(\mathbf{u} . \nabla) \mathbf{u}=-\nabla p, \\
\nabla \cdot \mathbf{u}=0 .
\end{gathered}
$$

In view of (6) there is a function $\phi$ such that $\mathbf{u}=\nabla^{\perp} \phi=(-\partial \phi / \partial y, \partial \phi / \partial x, 0)$; taking the curl of (5), with $\nabla \times \mathbf{u}=\Delta \phi$ then

$$
\frac{\partial}{\partial t} \Delta \phi+\nabla^{\perp} \phi \cdot \nabla \Delta \phi=0
$$

and we obtain (1) with $a=\lambda=0$. Notice that, at any time, the pressure is obtained by taking the divergence of (5), i.e.

$$
-\Delta p=\nabla .(\mathbf{u} . \nabla) \mathbf{u}=\nabla\left(\left(\nabla^{\perp} \phi . \nabla\right) \nabla^{\perp} \phi\right) .
$$


It is known that in the Euler case there are 'two' constants of motion, the energy $E$ and the vorticity $V_{f}$, respectively given by

$$
E=-\frac{1}{2} \int d^{2} x \phi \Delta \phi, \quad V_{f}=\frac{1}{2} \int d^{2} x f(\Delta \phi),
$$

$f$ being a continuous function; $V_{f}$ reduces to the enstrophy if $f=(\Delta \phi)^{2}$; if

$$
\Delta \phi=\rho \geqslant 0,
$$

$S=-\int d^{2} x \Delta \phi \ln \Delta \phi$ has been proposed for the entropy of the Euler flow (Montgomery et al. 1979). The energy and the enstrophy are the two constants of motion which survive standard truncations of the basic equation. The conservation of $E, V_{f}$ and also $S$ is ensured by the basic identity $\nabla^{\perp} \nabla=0$ applied to any function (Albeverio et al. 1979). Thus:

$$
\begin{gathered}
d E / d t=-\int d^{2} x \phi \nabla^{\perp} \phi \nabla \Delta \phi=\int d^{2} x\left(\nabla^{\perp} \phi \nabla \phi\right) \Delta \phi=0, \\
d V_{f} / d t=\int d^{2} x f^{\prime}(\Delta \phi) \nabla^{\perp} \phi \nabla \Delta \phi=-\int d^{2} x f^{\prime \prime}(\Delta \phi) \nabla^{\perp} \Delta \phi \nabla \Delta \phi=0 .
\end{gathered}
$$

In analogy with mean-field-type theories of point particles we investigate here approximated statistical states having the factorization property

$$
\langle\phi \phi\rangle \simeq\langle\phi\rangle\langle\phi\rangle
$$

and the problem is a more tractable one; in particular, for an approximated stationary statistical state we have from (3) that a class of solution is given by

$$
\Delta \phi=g([\phi]) \text { or }\langle\phi\rangle=h([\langle\Delta \phi\rangle]),
$$

where $g$ or $h$ is any functional of the corresponding variable. Thus

$$
\begin{aligned}
\left\langle\nabla^{\perp} \phi \nabla \Delta \phi\right\rangle & \simeq \nabla^{\perp}\langle\phi\rangle \nabla\langle\Delta \phi\rangle \\
& =\frac{1}{2}\left(\nabla^{\perp}\langle\phi\rangle \nabla\langle\phi\rangle \frac{\delta g}{\delta\langle\phi\rangle}+\nabla^{\perp}\langle\Delta \phi\rangle \nabla\langle\Delta \phi\rangle \frac{\delta h}{\delta\langle\Delta \phi\rangle}\right)=0
\end{aligned}
$$

since $\nabla^{\perp} \nabla=0$. (This has been already used in the proof of the conservation of energy and enstrophy.) In order to construct some approximated statistical states or to select a specific form of $g$ or $h$ in the case of stationary statistical states, we apply the principle of maximum entropy for continuous distributions and define the most probable states.

\section{Most probable states}

In the statistical mechanics of point particles, if $P$ is a probability measure on the phase space of the system, i.e. $\int P d \Omega=1$, then following Gibbs one can define a generalized $\mathscr{H}$ function given by:

$$
\mathscr{H}=-\int P \ln P d \Omega=\langle\ln P\rangle_{P} .
$$


Let $E(\phi)$ be the energy of the fluid and let $\mu(\phi)$ be a time-invariant probability measure for the Euler flow with density $d \mu(\phi)=P(\phi) d \phi$. If $\psi(t, \mathbf{x})$ is a solution of the Euler equation with $\psi(0, x)=\phi(x)$, then

$$
\langle E\rangle=\int E(\psi(t)) d \mu(\psi(t))=\int E(\phi) d \mu(\phi)
$$

Taking into account the conservation of the energy alone, the most probable statistical state is defined as the state which has the maximum entropy compatible with a given mean value of the constant of the motion $E$. Let $E$ be the only constant of the motion which is taken into account and let us denote by $\alpha$ the Lagrange multiplier associated with $E$; the principle of maximum entropy for $P(\phi)$ is given by

$$
\delta\left(-\int P(\phi) \ln P(\phi) d \phi+\alpha \int E(\phi) P(\phi) d \phi\right)=0 .
$$

Variational calculus (Hildebrand 1952) gives then the equilibrium canonical measure $\mu(\phi)=c e^{-E(\phi) \alpha}$.

Equilibrium measures as above have in fact been used earlier (Montgomery \& Fyfe 1976; Calinon \& Merlini 1979) to compute fluctuation spectra in various situations of inviscid two-dimensional magnetohydrodynamics and are stationary solutions of the Hopf equation.

Other computations may be done by considering different density in the entropy function. If the entropy of the fluid is taken as $S=-\int d^{2} x \rho \ln \rho$ (Montgomery et al. 1979) and introducing the Greens's function $G\left(x, x^{\prime}\right)$ associated with the equation $\Delta \phi=\rho$, the problem is to find a solution for the independent field $\rho$, such that

$$
\delta\left(-\int d^{2} x \rho \ln \rho+\alpha \int d^{2} x \rho(x) \int d^{2} x^{\prime} G\left(x, x^{\prime}\right) \rho\left(x^{\prime}\right)\right)=0 .
$$

Variational calculus gives then the solution

$$
\rho(\phi)=e^{-\alpha \phi}=\Delta \phi .
$$

In this case $\rho=f(\phi)$ and $\rho$ is a stationary solution of the Euler equation (1). The above equation bears analogies to some approximations in the statistical mechanics of point particles in the mean field limit (nonlinear Debye-Hückel equation) and is an analytically tractable case. Clearly the equation satisfied by the most probable state depends on the choice of the entropy function (see §4). We now apply this method to construct a statistical solution of the Euler flow having the factorization property discussed above.

Let $\alpha, \beta, \gamma$ be respectively the Lagrange multipliers associated with the conservation of the energy, the 'particle' number and the enstrophy of the Euler fluid and let us take as entropy the function $S=-\int d^{2} x\langle\rho\rangle \ln \langle\rho\rangle$. Then variational calculus gives

if

$$
\begin{gathered}
\delta\left(-\int d^{2} x\langle\rho\rangle \ln \langle\rho\rangle+\beta \int d^{2} x\langle\rho\rangle+\alpha \int d^{2} x d^{2} x^{\prime} G\left(x, x^{\prime}\right) \rho(x) \rho\left(x^{\prime}\right)\right. \\
\left.\left.+\gamma \int d^{2} x\langle\rho\}(x)\right\rangle^{2}\right)=0 \\
\Delta\langle\phi\rangle=\langle\rho\rangle=\exp (-\beta-\alpha\langle\phi\rangle-\gamma\langle\Delta \phi\rangle) .
\end{gathered}
$$


The functional $g$ in $(9)$ is thus selected. In particular, $\langle\rho\rangle$, the solution of (11), is an approximate statistical inhomogeneous state and is a stationary solution of the Euler equation.

As stated above, an analytically tractable case is given by setting $\gamma=0$ in (11); then

$$
\Delta\langle\phi\rangle=\exp (\beta-\alpha\langle\phi\rangle) .
$$

On a circular domain, the rotationally invariant solution of (12) is standard and given by

$$
\langle\phi\rangle(r)=a+b \ln \left(1+c\left(r / r_{0}\right)^{2}\right)
$$

with $\alpha b=2$ and $+8 c / \alpha r_{0}^{2}=e^{\beta-\alpha a}$, so that the two temperatures $\alpha, \beta$, are given by

$$
\begin{aligned}
& N=\int d^{2} x\langle\rho\rangle(x)=V \frac{e^{\beta-a \alpha}}{1+c}=V e^{\beta}(1+c), \\
& E=\int d^{2} x-\langle\phi\rangle \Delta\langle\phi\rangle=\frac{1}{\alpha} V e^{\beta}(1+c)\left(-1+\ln (1+c) \frac{(1+c)}{c}\right),
\end{aligned}
$$

where $V=\pi r_{0}^{2}$ is the volume of the domain and $\phi\left(r_{0}\right)=0$. Eliminating $\alpha$ and $\beta$ and introducing $\xi=16 \pi E / N^{2}$, the equation for $c$ is given by

$$
\frac{1}{2} \xi c^{2}=(1+c)^{2}\left(\ln (1+c)-\frac{c}{1+c}\right) .
$$

Equation (13) possesses always one solution in the range $-1<c<\infty$. Here negative temperature $\alpha$ means $c<0 ; \alpha>0$ if $c>0$ and the homogeneous case $c=0$ is obtained for $\xi=1$. The result illustrates the general effect of a global constant of the motion like $\xi$ on the structure of the statistical density profile for the field $\phi$. From (8), we may now compute the pressure and obtain

$$
p(r)=\frac{1}{\alpha} \frac{N}{V} \frac{(1+c)}{1+c\left(r / r_{0}\right)^{2}}+K .
$$

The constant $K$ may be chosen such that at the wall $p\left(r_{0}\right)=\alpha^{-1} \rho\left(r_{0}\right)$. This concludes the analysis of (11) in the special case $\gamma=0$.

In the case of two types of 'charges' $\left(\rho_{1}(x), \rho_{2}(x) \geqslant 0\right)$, such that

$$
\Delta\langle\phi\rangle=\left\langle\rho_{1}\right\rangle-\left\langle\rho_{2}\right\rangle
$$

equation (11) is easily generalized to

$$
\Delta\langle\phi\rangle=\sinh (\alpha\langle\phi\rangle+\beta+\gamma\langle\Delta \phi\rangle)
$$

On the other hand, if one species of 'charge' is represented by a homogeneous neutralizing background $\left(\rho_{2}=1\right)$, we obtain

$$
\Delta\langle\phi\rangle=\exp (\alpha\langle\phi\rangle+\beta+\gamma\langle\Delta \phi\rangle)-1
$$

in suitable units. To compare with previous work on systems of point particles, we notice that as $\gamma \rightarrow 0$, and depending on the signature of $\alpha$, equation (15) reduces respectively to the shielding or anti-shielding Poisson equation for twocomponent plasmas (Choquard 1976; Calinon \& Merlini 1979); it also reduces to the guiding centre equation (Montgomery \& Joyce 1974); if $\gamma=0$, (11) reduces to the one considered in a recent treatment of a system of point vortices (Lundgren $\&$ Pointin 1977) and possesses a wide range of solutions (Williamson 1977). 


\section{Drift-wave turbulence and two-dimensional magnetofluids}

We now apply the method to some two-dimensional and pseudo-three-dimensional models.

\subsection{Drift-wave equation}

We begin with a model of drift-wave turbulence proposed recently (Hasegawa \& Mima 1978); the evolution of the potential $\phi$ is governed by (1) with $a \equiv 0$. Notice that such an equation is a nonlinear form of the general drift equation (Biskamp \& Horton 1980) recently studied. The three constants of motion which come into play here are given by

$$
\begin{aligned}
& S_{1}=\int d^{2} x\left(\Delta \phi-\lambda^{2} \phi\right) \\
& S_{2}=\int d^{2} x\left(\Delta \phi-\lambda^{2} \phi\right) \phi \\
& S_{3}=\int d^{2} x\left(\Delta \phi-\lambda^{2} \phi\right) \Delta \phi
\end{aligned}
$$

The natural candidate for the entropy function is given by $S=-\int d^{2} x \rho \ln \rho$ since $\rho=\Delta \phi-\lambda^{2} \phi$ plays a role analogous to the charge density in the Euler case treated in $\S 3$. (We suppose $\rho \geqslant 0$.) Then $\phi=\int d^{2} x G\left(x, x^{\prime}\right) \rho\left(x^{\prime}\right)$ where $G\left(x, x^{\prime}\right)$ is the Green's function of the equation $\left(\Delta-\lambda^{2}\right) \phi=\rho$; let $\beta, \alpha$ and $\gamma$ be the three temperatures associated with $S_{1}, S_{2}, S_{3}$; applying the principle of maximum entropy we obtain

$$
\Delta \phi=\lambda^{2} \phi+\exp (\beta+\alpha \phi+\gamma \Delta \phi) .
$$

The above equation differs from (11) by the presence of the screening term $\lambda^{2} \phi$; clearly the procedure is not unique; in fact another possibility is to associate the entropy $S^{\prime}=-\int d^{2} x \Delta \phi \ln \Delta \phi$; this would result in a non-local Poisson equation given by

$$
\Delta \phi=\exp \left[\beta\left(\lambda^{2}-1\right)+\alpha\left(\lambda^{2} \psi-\phi\right)+\gamma\left(\lambda^{2} \phi-\Delta \phi\right)\right]
$$

with $\Delta \psi=\phi$, which still reduces to (11) as $\lambda \rightarrow 0$; (17) seems to us more appropriate and the numerical solutions will be reported elsewhere.

\subsection{Two-dimensional magnetohydrodynamics}

As $\lambda \rightarrow 0,(1)$ and (2) describe two-dimensional magnetohydrodynamic flows. We limit ourselves to discussing the pure toroidal case where $a=B$; the constants of motion are known (Calinon \& Merlini 1979); they are the energy and the enstrophy of the fluid, the magnetic energy and a quantity which plays the same role as the cross-helicity in the pure poloidal case. They are

$$
S_{1}=\frac{1}{2} \int d^{2} x \phi \Delta \phi, \quad S_{2}=\frac{1}{2} \int d^{2} x(\Delta \phi)^{2}, \quad S_{3}=\frac{1}{2} \int d^{2} x B^{2}, \quad S_{4}=\int d^{2} x \Delta \phi B
$$


Let $\alpha, \gamma, \delta, \beta$ be the four temperatures associated with $S_{i}, i=1, \ldots, 4$. The entropy functional now takes the form

$$
\begin{aligned}
F=- & \int d^{2} x \rho_{1} \ln \rho_{1}-\int d^{2} x \rho_{2} \ln \rho_{2}-\int d^{2} x \xi(B) \ln \xi(B) \\
& +\frac{1}{2} \alpha \int d^{2} x \phi\left(\rho_{1}-\rho_{2}\right)+\frac{1}{2} \gamma \int d^{2} x\left(\rho_{1}-\rho_{2}\right)^{2}+\frac{1}{2} \delta \int d^{2} x B^{2}+\beta \int d^{2} x\left(\rho_{1}-\rho_{2}\right) B,
\end{aligned}
$$

where we assume two types of 'charges' such that $\Delta \phi=\rho_{1}-\rho_{2}\left(\rho_{i} \geqslant 0\right)$ and $\xi(B)$ is some functional of $B$. We will limit ourselves to the case in which the magnetic field lines point only in one direction. Then $\rho_{1}, \rho_{2}, B$ may be taken as the three independent fields and a natural choice is $\xi(B)=B$; in this case $\delta F=0$ if

$$
\begin{gathered}
\Delta \phi=\sinh (\alpha \phi+\gamma \Delta \phi+\beta B), \\
B=\exp (\delta B+\beta \Delta \phi) .
\end{gathered}
$$

If we choose the scalar $\Delta B=\omega$ as an independent field instead of $B$, then it is natural to set $\xi([B])=\xi(\Delta B)=\omega$; this choice gives rise as before (\$4.1) to a non-local Poisson equation since, with $\Delta B=\omega, B=\int d^{2} x^{\prime} G\left(x, x^{\prime}\right) \omega\left(x^{\prime}\right)$, and $\delta F=0$ if

$$
\begin{gathered}
\Delta \phi=\sinh (\alpha \phi+\gamma \Delta \phi+\beta B), \\
\omega=\Delta B=\exp (\beta \phi+\delta \psi)
\end{gathered}
$$

with $\Delta \psi=B$; in the particular case where $\beta=0$, the profile for $\psi$ is given by the solution of a generalized nonlinear biharmonic equation

$$
\Delta(\Delta \psi)=e^{\delta \psi} .
$$

An analytical solution is not available and numerical work is necessary. The situation is different if we consider the 'mixed' choice such that $\Delta B=\omega$ is regarded as independent field but where the entropy function is still given by $\xi([B])=B$, i.e. $\xi([B])=B=\int d^{2} x G\left(x, x^{\prime}\right) \omega\left(x^{\prime}\right)$. Let $G\left(x, x^{\prime}\right)$ be the Green's function associated with the equation $\Delta B=\omega$. Variational calculus gives (19) as before while (20) transforms into

$$
B=\exp (\beta \phi+\delta \psi)
$$

with $\Delta \psi=B$. In the particular case $\beta=0,(24)$ is still analytically tractable as in the Euler case $(\$ 3)$ and the statistical profile for the magnetic field follows from the solution for $\psi$ given by

$$
\psi(r)=\frac{2}{\delta} \ln \frac{1+c}{1+c\left(r / r_{0}\right)^{2}} .
$$

with $(1+c)^{2}=-8 c / \delta r_{0}^{2}$ and at the boundary $\psi\left(r_{0}\right)=0$. Then

$$
B(r)=\frac{(1+c)^{2}}{\left(1+c\left(r / r_{0}\right)^{2}\right)^{2}}
$$

The magnetic energy is given by $\mathscr{B}=\frac{1}{2} \int d^{2} x B^{2}=V(1+c)$ and the temperature $\delta$ by

$$
\delta=\frac{8}{r_{0}^{2}} \frac{(1-\mathscr{B} / V)}{(\mathscr{B} / V)^{2}}
$$


The magnetic field is thus obtained as

$$
B(r)=\left(\frac{\mathscr{B}}{V}\right)^{2} \frac{1}{\left[1+\left(r / r_{0}\right)^{2}\right.} \frac{(\mathscr{B} / V-1)]^{2}}{.}
$$

The above result still illustrates the general role of global constants such as $\mathscr{B} / V$; if the energy density $\mathscr{B} / V$ is greater than $1(\delta<0)$, the profile for $B$ is monotonic decreasing away from the central region, while if $\delta>0$ it is increasing. The case $\mathscr{B} / V=1$ corresponds to the infinite temperature limit $1 / \delta \rightarrow \infty$, with the constant magnetic field normalized to $B=1$. This concludes the discussion of the toroidal case for $\beta=0$. The poloidal case where $\nabla \times \mathbf{a}=\mathbf{B}$ has been treated previously (Montgomery et al. 1979); in that case, a particular analytical solution for $a$ has already been found and the profile for $a$ is given by (12) with $\phi$ replaced by $a$.

\subsection{Model of an electromagnetic filamentation instability}

We now consider a pseudo-three-dimensional model proposed recently (Montgomery \& Liu 1980) describing the nonlinear evolution of an electromagnetic filamentation instability which is a variety of the Weibel instability (Weibel 1959); approximated energy spectra for the homogeneous situation have been computed; nevertheless, statistical stationary inhomogeneous states have not been investigated in a configuration space formulation of the problem even approximately. The principle of maximum entropy provides such a treatment. Let us start with the equation governing the evolution of the two-beam density, potentials and velocities given by (Montgomery \& Liu 1980):

$$
\begin{gathered}
\partial n_{ \pm} / \partial t+\nabla \cdot\left(n_{ \pm} \cdot \mathbf{v}_{ \pm}\right)=0 \\
\partial \mathbf{v}_{ \pm} / \partial t+\left(\mathbf{v}_{ \pm}^{\perp} \cdot \nabla\right) \mathbf{v}_{ \pm}^{\perp}=(e / m)\left(\nabla \phi-v_{z}^{\perp} \nabla A\right) \\
\Delta \phi=-4 \pi e\left(2 n_{0}-n_{+}-n_{-}\right)=\rho \\
\Delta A=(4 \pi e / c)\left(n_{+} v_{z}^{+}+n_{-} v_{z}^{-}\right)=\gamma
\end{gathered}
$$

where $\mathbf{v}_{ \pm}=\mathbf{v}_{ \pm}^{\perp}+\mathbf{e}_{z} v_{z}^{ \pm}$.

Notice that the velocities of the two beams in the $z$ direction have been given as $v_{z}^{ \pm}= \pm u+e A / m c$; the superscript $\perp$ means velocity component in the $(x, y)$ plane perpendicular to the $z$ axis and $2 n_{0}$ is the constant charge density of a neutralizing homogeneous background. All quantities vary only in the $(x, y)$ directions. Since the conservation of momentum in the $z$ direction has been taken into account, the only constant of motion which comes into play here is the energy:

$$
\begin{aligned}
\epsilon & =\int d^{2} x \frac{m}{2}\left(n_{+} \mathbf{v}_{+}^{2}+n_{-} \mathbf{v}_{-}^{2}\right)+\frac{1}{8 \pi} \int d^{2} x\left((\nabla \phi)^{2}+(\nabla A)^{2}\right) \\
& =\int d^{2} x\left(\frac{m}{2}\left(n_{+} \mathbf{v}_{+}^{2 \perp}+n_{+} u^{2}+n_{-} \mathbf{v}_{-}^{2 \perp}+n_{-} u^{2}\right)-\frac{\phi \Delta \phi}{8 \pi}+\frac{u e A}{2 c}\left(n_{+}-n_{-}\right)\right) .
\end{aligned}
$$

It should be noticed that the equation for $\phi$ is linear in the densities unlike that for $A$. In fact,

$$
\Delta A-\frac{\omega_{c}^{2}}{c^{2}} A=\gamma_{1}+\gamma_{2}=\frac{4 \pi e u}{c}\left(n_{+}-n_{-}\right)+\frac{4 \pi e^{2} A}{m c^{2}}\left(n_{+}+n_{-}-2 n_{0}\right)
$$


where $\omega_{c}^{2}=8 \pi n_{0}^{2} e / m$; if $e A / m c \ll u$ (which is the condition that the fractional slowing down of the electron streams shall be small) then the nonlinear term may be neglected, (28) becomes linear and the problem is a more tractable one. Then

$$
\epsilon \simeq \epsilon_{1}=\int d^{2} x\left(\frac{m}{2}\left[n_{+}\left(\mathbf{v}_{+}^{2 \perp}+u^{2}\right)+n_{-}\left(\mathbf{v}_{-}^{2 \perp}+u^{2}\right)\right]-\frac{\phi \Delta \phi}{8 \pi}+\frac{A \gamma_{1}}{8 \pi}\right)
$$

We look at approximated states having the factorization property

$$
\left\langle n_{ \pm} \mathbf{v}_{ \pm}^{2 \perp}\right\rangle \simeq\left\langle n_{ \pm}\right\rangle\left\langle\mathbf{v}_{ \pm}^{2 \perp}\right\rangle
$$

Part of an entropy functional may be given explicitly in terms of the basic independent variables $n_{+}, n_{-}$(which form their definition are non-negative) and $\left\langle\mathbf{v}_{ \pm}^{2 \perp}\right\rangle$ as

$$
F=-\int d^{2} x\left(n_{+} \ln n_{+}+n_{-} \ln n_{-}\right)-\int d^{2} x\left(f\left(\left\langle\mathbf{v}_{+}^{2 \perp}\right\rangle\right)+f\left(\left\langle\mathbf{v}_{-}^{2 \perp}\right\rangle\right)-\alpha \epsilon_{1} .\right.
$$

$f\left(\langle v\rangle_{ \pm}^{2 \perp}\right)$ remains unspecified. Then with

$$
\phi=\int d^{2} x G_{1}\left(x, x^{\prime}\right)\left(n_{+}+n_{-}\right) \quad \text { and } \quad A=\int d^{2} x G_{2}\left(x, x^{\prime}\right)\left(n_{+}-n_{-}\right)
$$

where $G_{1}, G_{2}$ are respectively the Green's function associated with $(27 c)$ and $(27 d)$ with $\gamma_{2}=0$, variational calculus may be performed for $n_{ \pm}$, yielding $\delta F=0$ if

$$
n_{ \pm}=\exp \left[\alpha\left(\frac{1}{2} m\left(\left\langle\mathbf{v}_{ \pm}^{2 \perp}\right\rangle+u^{2}\right)-\phi \pm(u / c) A\right)\right]
$$

$\left\langle\mathbf{v}_{ \pm}^{2 \perp}\right\rangle$ remains unspecified but, since we look at a stationary state, the equation $\left\langle\left(\mathbf{v}_{ \pm}^{\perp} . \nabla\right) \mathbf{v}_{ \pm}^{\perp}\right\rangle=(e / m)\left\langle\nabla \phi-v_{z}^{ \pm} \nabla A\right\rangle$ should be satisfied so that $\left\langle\mathbf{v}_{ \pm}^{2 \perp}\right\rangle$ may be computed. Here we restrict the analysis to the case where cubic terms in the expression for $\epsilon_{1}$ may be neglected, i.e.

$$
\frac{1}{2} m n_{ \pm}\left(\left\langle\mathbf{v}_{ \pm}^{2 \perp}\right\rangle+u^{2}\right)=\frac{1}{2} m\left(n_{0}+n_{ \pm}\right)\left(\left\langle\mathbf{v}_{ \pm}^{2 \perp}\right\rangle+u^{2}\right) \simeq \frac{1}{2} m n_{0}\left(\left\langle\mathbf{v}_{ \pm}^{2 \perp}\right\rangle+u^{2}\right)
$$

writing

$$
\delta n_{ \pm}=n_{ \pm}-n_{0}
$$

Then the equations for $\phi$ and $A$ (in appropriate units where $n_{0}=1$ ) are simplified further:

$$
\begin{gathered}
\Delta \phi=4 \pi e\left(2 \cosh \left(\alpha \frac{u}{c} A\right) e^{-\alpha \phi}-2\right), \\
\Delta A-\frac{\omega_{c}^{2}}{c^{2}} A=4 \pi e \frac{u}{c} 2 \sinh \left(\alpha \frac{u}{c} A\right) e^{-\alpha \phi} .
\end{gathered}
$$

Equations (30) and (31) are to be solved with appropriate boundary conditions and $\alpha$ is to be determined, by requiring that the total energy has a given value $\epsilon_{1}$. The above equations may possess a rich variety of solutions depending on the values of $\epsilon_{1}$, and numerical computations are in progress. To conclude this work, we shall treat in detail a one-dimensional model without neglecting cubic terms. 


\section{One-dimensional model}

We shall treat the one-dimensional one-fluid model described by the equations

$$
\begin{gathered}
\frac{\partial \rho}{\partial t}+\frac{\partial}{\partial x}(\rho v)=0, \\
\frac{\partial v}{\partial x}+v \frac{\partial v}{\partial x}=-E=\nabla \phi, \quad \Delta \phi=\rho .
\end{gathered}
$$

For the sake of simplicity we will assume only one species of charges $(\rho(x) \geqslant 0)$; this has the advantage that an analytical solution may be given. The constants of motion are here the total energy $\epsilon=\frac{1}{2} \int d x\left(\rho v^{2}+E^{2}\right)$ and the total charge $N=\int d x \rho(x)$. (Strictly speaking $\epsilon=\epsilon_{1}+\epsilon_{2}$, where $\epsilon_{1}$ and $\epsilon_{2}$ are both constants of motion; $\epsilon_{1}$ is the energy for the mode $\mathbf{k}=0, \epsilon_{2}$ that of the modes $\mathbf{k} \neq 0$ in a Fourier space formulation of the problem; we will nevertheless consider here only a temperature $\alpha$ associated with $\epsilon$ and $\beta$ associated with $N$.) $\rho(x)$ and $v(x)$ are the two independent fields. Notice that $E$ contains cubic terms like $\rho v^{2}$, which are usually neglected in a Fourier space formulation for similar models (Fyfe \& Montgomery 1978). Here we will not discard cubic terms, but look at states such that $\langle v(x)\rangle=c_{1}\left(c_{1}\right.$ constant $),\left\langle v^{2}(x)\right\rangle$ is not constant and such that $\left\langle\rho v^{2}\right\rangle=\langle\rho\rangle\left\langle v^{2}\right\rangle$. Using the condition of stationary states such that

$$
\left\langle\frac{1}{2} v^{2}\right\rangle=\langle\phi\rangle+c_{2}
$$

(equation $(32 b), c_{2}$ constant), we may eliminate the velocity field and consider a functional for $\rho(x)$ alone:

$$
F=-\int d x \rho(x) \ln \rho(x)+\frac{1}{2} \alpha \int d x\left(-\phi \rho+2 \rho\left(\phi+c_{2}\right)\right)+\beta \int d x \rho(x) .
$$

Variational calculus gives then the equation

$$
\Delta \phi=\exp \left(\beta+\alpha c_{2}+\alpha \phi\right)
$$

which still possesses an analytical solution; in fact $\rho(x)$ is a static 'soliton' given by

$$
\rho(x)=-\frac{2}{\alpha}\left(\frac{k}{L}\right)^{2} \frac{1}{\cosh ^{2}(k x / L)}
$$

since $\phi(x)=(2 / \alpha) \ln [\cosh k / \cosh (k x / L)]$ is the solution of $(33)$ with $\phi( \pm L)=0$, and $V=2 L$ is the volume of the domain. Moreover

$$
-\frac{2}{\alpha}\left(\frac{k}{L}\right)^{2}=\exp \left(\beta+\alpha c_{2}\right) \cosh ^{2} k
$$

Notice that $\alpha$ may be positive ( $k$ pure imaginary) or negative ( $k$ real). The two additional relations which allow us to $\mathrm{fix} \alpha, \beta$ and $k$ are given by

$$
\begin{aligned}
N & =\int_{V} d x \rho(x)=\exp \left(\beta+\alpha c_{2}\right) \cosh ^{2} k \frac{\tanh k}{k} V \\
\epsilon & =\int_{V} d x\left(\rho \phi+c_{2} \rho\right)=c_{2} N+N \frac{k}{\tanh k}\left(1-\frac{\tanh k}{k}\right) \frac{1}{\alpha} .
\end{aligned}
$$


Eliminating $\alpha$ and $\beta, k$ is finally given by

$$
c_{2}-\frac{\epsilon}{N}=\frac{N^{2}}{8 \rho_{0}}\left(\frac{1}{\tanh ^{2} k}-\frac{1}{k \tanh k}\right)
$$

where $\rho_{0}=N / V$. Equation (38) possesses real or pure imaginary solutions for $k$, depending on the values of the parameters $c_{2}, N$ and $\epsilon$. We will discuss the particular case where the potential energy is equal to the kinetic energy; in this case $c_{2} N=\frac{3}{2} \epsilon$. Since $\left\langle\frac{1}{2} v^{2}\right\rangle=\langle\phi\rangle+c_{2}$ should be non-negative, the additional inequality which $k$ has to satisfy is given by

$$
c_{2}+(2 / \alpha) \ln [\cosh k / \cosh (k x / L)] \geqslant 0, \quad \forall|x|<L .
$$

Introducing $\epsilon^{\prime}=\epsilon 4 \rho_{0} / N^{3}$, one then has to solve the equation

with the constraint

$$
\epsilon^{\prime}=\frac{1}{\tanh ^{2} k}-\frac{1}{k \tanh k}
$$

$$
\epsilon^{\prime} \geqslant \frac{2}{3} \frac{\ln (\cosh k)}{k \tanh k}
$$

From the numerical solution, if $k$ is real, $\epsilon^{\prime}(k)$ is monotonic increasing in the range $k<0.2$; if $k=i \bar{k}$ is pure imaginary, $\epsilon^{\prime}(\bar{k})$ is monotonic decreasing in the same domain. $\bar{k}<0.2$ and inequality (40) is fulfilled in the same range for $k$ either real or pure imaginary. $\epsilon^{\prime}(k \rightarrow 0)=\epsilon^{\prime}(\bar{k} \rightarrow 0)=\frac{1}{3} ; \epsilon^{\prime}(k=0.2) \simeq 1.2$ and $\epsilon^{\prime}(\bar{k}=0 \cdot 2) \simeq 0 \cdot 06$. Thus, for a given $\epsilon^{\prime}>0$, such that $0.06<\epsilon^{\prime}<1 \cdot 2$, there is a unique solution for $\alpha, \beta, k, \bar{k}$. The density profiles are given by

$$
\begin{aligned}
& \rho(x)=\rho_{0} \frac{k}{\tanh k} \frac{1}{\cosh ^{2}(k x / L)}, \\
& \rho(x)=\rho_{0} \frac{\bar{k}}{\tan \bar{k}} \frac{1}{\cos ^{2}(k x / L)} .
\end{aligned}
$$

In the first case $(k<0$ and $\alpha<0)$, the density is peaked at the origin while if $k>0$ and $\alpha>0$ the density is greater at the boundary. As $k, \bar{k} \rightarrow 0, \epsilon^{\prime}=\frac{1}{3}$ and $\rho(x)=\rho_{0}$.

\section{Conclusion}

A method has been followed for selecting approximate inhomogeneous timeasymptotic statistics of general two-dimensional magnetofluids, whose states have maximum entropy and are associated with the law of conservation of some global constants of the motion. The statistical profiles for the fields have been found to be the solution of a rich class of nonlinear generalized Poisson equations, which are simple in structure but which will in general require a numerical solution, and they are similar to mean field equations in the statistical mechanics of point particles. Some limiting cases have been discussed analytically and in all cases it has been shown that a qualitative change in the structure of the profiles in a finite container arises as some parameters, related to the constants of the motion, exceed certain critical values. Work is in progress on the numerical solution of some of the non-trivial nonlinear equations which appeared in this work. 
It is a pleasure to thank both Professor Sergio Albeverio and Professor Ken Golden for their kind hospitality, for reading the manuscript, and for making interesting remarks. The financial support of the Fonds National Suisse and the Deutsche Forschungsgemeinschaft is gratefully acknowledged.

\section{REFERENCES}

Albeverio, S. \& Høegh-Krohn, R. 1979 Preprint.

Albeverio, S., Ribeiro de Faria, M. \& Høegh-Krohn, R. 1979 J. Stat. Phys. 20, 585. Biskamp, D. \& Horton, W. 1980 Phys. Lett. 75 A, 359.

Calinon, R. \& Merlini, D. 1979 J. Plasma Phys. 22, 385.

Choquard, Ph. 1975 Research note Princeton-EPFL-Lausanne: On a class of chargeordered states in simple Coulomb system.

Fyfe, D. \& Montgomery, D. 1978 Phys. Fluids, 21, 317.

Hasegawa, A. \& Mima, K. 1978 Phys. Fluids, 21, 87.

Hildebrand, F. B. 1952 Methods of Applied Mathematics. Prentice Hall.

Lundaren, T. S. \& Pointin, Y. B. 1977 J. Stat. Phys. 17, 323.

Montgomery, D. \& Fyfe, D. 1976 J. Plasma Phys. 16, 181.

Montgomery, D. \& Liv, C. S. 1979 Phys. Fluids, 22, 866.

Montgomery, D. \& Joyce, G. 1974 Phys. Fluids, 17, 1139.

Montgomery, D., Turner, L. \& Vahata, G. 1979 J. Plasma Phys. 21, 239.

Montgomery, D. \& Vahala, G. 1979 J. Plasma Phys. $21,71$.

Salmon, R. et al. 1976 J. Fluid Mech. 75, 691.

Weibel, E. S. 1959 Phys. Rev. Lett. 2, 83.

Williamson, J. H. 1977 J. Plasma Phys. 17, 85. 